\section{Multilayered Controlled Released Topical Patch Containing Tetracycline for Wound Dressing}

\section{Abstract}

Proficient antibiotic drug delivery plays a key role in treating infections. In this article we prepared an electrospun wound dressing which releases Tetracycline in a timed and dose controlled matter for one week. In order to fabricate a wound dressing which can release Tetracycline in a longer duration and at an effective therapeutic level we designed a bilayer electrospun patch made of polyvinyl alcohol (PVA), polycaprolacton (PCL), and coated the whole system with chitosan. The SEM micrograph showed the dependent relationship of the morphology of the nanofibers on the Tetracycline concentration, and the addition of PCL electrospun layer decreased the pore size and water uptake rate. However, it was found that coating PVA/PCL bilayered electrospun sheet with chitosan increased the water uptake rate, which is an important property in biomedical applications. According to the FTIR results, chemical composition of the scaffolds did not change during the fabrication process. Mechanical properties of the samples were assessed, and the results revealed that the chitosan coating improved the tensile strength of the electrospun layers. Furthermore, based on in vitro release tests, it was found that bilayered electrospun sheets coated with chitosan controlled drug release efficiently. In addition, the antibacterial tests performed in vitro showed stronger antibacterial activity on S. aureus cultures for PVA/PCL/Chitosan in comparison to uncoated samples.

Keywords: Chitosan, polycaprolacton, polyvinyl alcohol, electrospining, Tetracycline, control released drug delivery

\section{Gita Kiaee" , Mahbobe Etaat" , Bita Kiaee ${ }^{2}$, Shadi Kiaei ${ }^{3}$ and Hamid Akbari Javar ${ }^{1}$

\author{
1 Tehran university of Medical \\ Sciences,Tehran, Iran \\ 2 Islamic Azad University of Medical Science, \\ Tehran, Iran \\ 3 Portland state university, USA
}

\section{Corresponding author: Gita Kiaee}

\section{Đ gkiaee@gmail.com}

Doctor of pharmacy,Tehran university of Medical Sciences,Tehran, Iran.

Tel: +98 (21) 88896692

Citation: Kiaee G, Etaat M, Kiaee B, et al. Multilayered Controlled Released Topical Patch Containing Tetracycline for Wound Dressing. J In Silico In Vitro Pharmacol. 2016, 2:2.

\section{Introduction}

Wound infection is a prevalent medical problem especially in hospital settings and it needs to be address properly [1, 2]. Aside from the infection itself, there are other altercations to wound infections. Wound infections also increases the total cost of health care delivery due to longer duration of hospitalization, and the additional treatments required to properly treat the infection. Topical administration of antibiotics provides higher local concentration of antibiotics with a lower systemic level in comparison to systemic administration which can lead to immediate effects of the antibacterial [3]. Several clinical studies showed that various antibiotics such as Tetracycline, Cefazolin, Vancomycin, Neomycin and Ampicillin can be administered topically to eradicate bacteria effectively [4]. By designing a drug delivery system which can effectively administer constant drug release we can enhance the efficacy of wound dressings and prevent future infections. In addition the new drug delivery system will increase patient compliance by reducing the time required to change wound dressing.

Based on pharmacodynamic characteristics, antibiotics are classified by three categories [5] concentration-dependent, timedependent, and bacteriostatic groups. In references to these categories, optimal antibacterial effects are achieved according to the time and concentration of the drugs. Concentrationdependent antibiotics optimal bactericidal effects have been seen in concentrations above 10 times the minimum inhibitory concentration (MIC). For time dependent antibacterial, drug concentrations need to be maintained above the MIC during dose interval. However, bacteriostatic antibiotics are a composition 
of both concentration-dependent and time-dependent group characteristics. Furthermore, the antibacterial activity of bacteriostatic antibiotics depends on the continuous release of a high local concentration for an extended time interval [6].

Different methods exist for fabricating a wound dressing. Among the various methods, electro-spinning shows great promise due to its highly porous structure and a very large surface area-tovolume ratio. These two factors of electrospun fibrous mats allows for a better drug penetration and release rate $[7,8]$. Moreover, the electrospun nanofibers can serve as the substrate for tissue regeneration by promoting cell adhesion, spreading, and proliferation [9]. Electrospining utilizes a high electric force to generate a polymer between a solution contained in a syringe with a capillary tip and a target. Different parameters effects fiber diameter such as the electric voltage, polymer solution concentration, and solvent composition. The process can be adjusted to control the fiber diameter [10].

In order to construct a durable and effective drug delivery system, polyvinyl alcohol (PVA), poly caprolactone (PCL) and chitosan were used. The approach to address the improvement of drug release and physical properties of wound dressing was to produce a multilayered nanofibrous sheet with different distinct layers. Each layer will play distinct a function. The first layer will act as the drug reservoir and contain PVA and Tetracycline. PVA has attracted much attention for wound dressing because PVA has demonstrated better wound sanitation and an increased tissue regrowth compared to other materials [11]. However there are some problems with the practical application of PVA including poor mechanical strength and the water evaporation of the polymer which can lead to the stiffening of the hydrogel and cause tissue damage [12]. Moreover, the chemical crosslinking agent residue such as glutaraldehyde has demonstrated to have adverse effects on health $[13,14]$. In addition, physical crosslinking such as thermal treatment affects drug and may cause the degradation of drug [15]. With only a PVA layer, a wound dressing would need to be changed frequently, during which the renewed skin has a higher risk of becoming damaged again.

The second layer of the new nanofibrous system will contain PCL which will act as the barrier to control the release profile of the drug, enhance the mechanical strength of PVA, and prevent water evaporation from the hydrogel. $\mathrm{PCL}$ is the great candidate for acting as the barrier layer due to its hydrophobic nature and also good mechanical properties [16]. In the Zamani et al. [8] study, a PCL electrospun sheet provided a sustainable drug release system which lasted for 19 days [8]. Similarly, the Zahedi et al. [17] showed that a PVA/PCL (80/20) electrospun sheet had a much better drug release rate for Tetracycline as a drug model compared with cast film samples containing the same drug [17].

In order for the wound dressing to release Tetracycline in longer durations and at an effective therapeutic level, we designed a bilayer electrospun fiber coated with chitosan. Chitosan's chemical properties act similarity to the glycosaminoglycans in the extracellular matrix (ECM), and chitosan has a morphological proximity to fibrous collagen structures in the ECM on the nanometer scale [18]. Chitosan also enhances the function of inflammatory cells such as macrophages, polymorphonuclear leukocytes, and fibroblast which lead to the granulation and organization promotion in wound area [19]. In addition, the Kang et al. [18] study conducted on mice found that PVA nanofiber dressing coated with chitosan was effective in early stages of wound healing [18]. Therefore, on the basis of wound healing, acceleration, and antimicrobial activity, a surface coated with chitosan was considered as an effective approach. Additionally, chitosan can reduce the drug release rate, and give a controllable release system which can improve therapeutic efficacy and safety of the antibiotic. This is done by delivering the drug at the rate dictated by the need of the physiological environment over the treatment period [20]. Considering the chemical and physical properties of chitosan, in this study as shown in (Figure 1), we coated the nanofirbrous bilayer PVA and PCL with chitosan to deliver Tetracycline to the site of infection at a controlled rate.

\section{Materials and Methods}

\section{Material}

Polyvinyl alcohol (PVA) (trade name, GH-17RGOHSENOL, a whiteyellowish powder, viscosity of $4 \%$ solution $=30 \mathrm{mPa} . \mathrm{s}$, melting point $180^{\circ} \mathrm{C}$ ) was purchased from NIPPON GOHSEI Co., Japan. Poly ( $\varepsilon$-caprolactone) (PCL) (biodegradable, weight average molecular weight $90 \mathrm{~kg} / \mathrm{mol}$ ) was obtained from Sigma-Aldrich Co., Pilsburg, The Netherlands. Tetracycline hydrochloride, an antibiotic (99.99\% purity, yellow powder) was supplied by Merck, Germany. Medium molecular weight Chitosan was bought from Chitoclear-I and All the other chemicals were analytical reagent grades and were used without further purification.

\section{Preparation of multilayer drug-loaded electrospun PVA and PCL}

A predetermined weight of PVA powder was dissolved in distilled water at $80^{\circ} \mathrm{C}$, stirred for about $2 \mathrm{~h}$, and finally a $10 \%(\mathrm{w} / \mathrm{v})$ solution of PVA in distilled water was obtained. After cooling the PVA solution to room temperature, pre-weighed Tetracycline was added to PVA solution to get a $3 \%(\mathrm{w} / \mathrm{v})$ drug concentration. The mixture was stirred for about $30 \mathrm{~min}$. An electrospinning device model (eSpinner NF-COEN/II) Asian Nanostructures Technology Co. was used for preparation of the samples. The electrospinning conditions for PVA sample were: solution flow rate $=0.8 \mathrm{~mL} / \mathrm{h}$, voltage $=19.5 \mathrm{kV}$, gauge needle $=0.7 \mathrm{~mm}$, and the distance between the needle tip to the rotational collector of $10 \mathrm{~cm}$. Similarly, weighed granules of $P C L$ was dissolved in $7 / 3(\mathrm{v} / \mathrm{v})$ solution of chloroform/DMF in order to get a $10 \%(\mathrm{w} / \mathrm{v})$ concentration and stirred for about $1 \mathrm{~h}$. The elecrospinning conditions for PCL sample were as follows: solution flow rate $=0.45 \mathrm{~mL} / \mathrm{h}$, voltage $=19.5 \mathrm{kV}$, gauge needle $=0.7 \mathrm{~mm}$, and distance between the needle tip to the rotational collector $=14.5 \mathrm{~cm}$. Two layers of nanofiber mats was prepared in the $24 \mathrm{~h}$ time interval consecutively, however, in order to overlay a second layer the first layer had to have dried completely.

Preparation of PVA/PCL nanofibrous matrix coated with chitosan: The chitosan solution ( $1.0 \mathrm{wt} \%$ and $0.5 \%$ ) for coating the PVA/PCL nanofibrous matrix was prepared by dissolving chitosan powder in an aqueous $1 \%$ acetic acid solution at room temperature. Then the PVA/PCL nanofibrous matrix was coated 


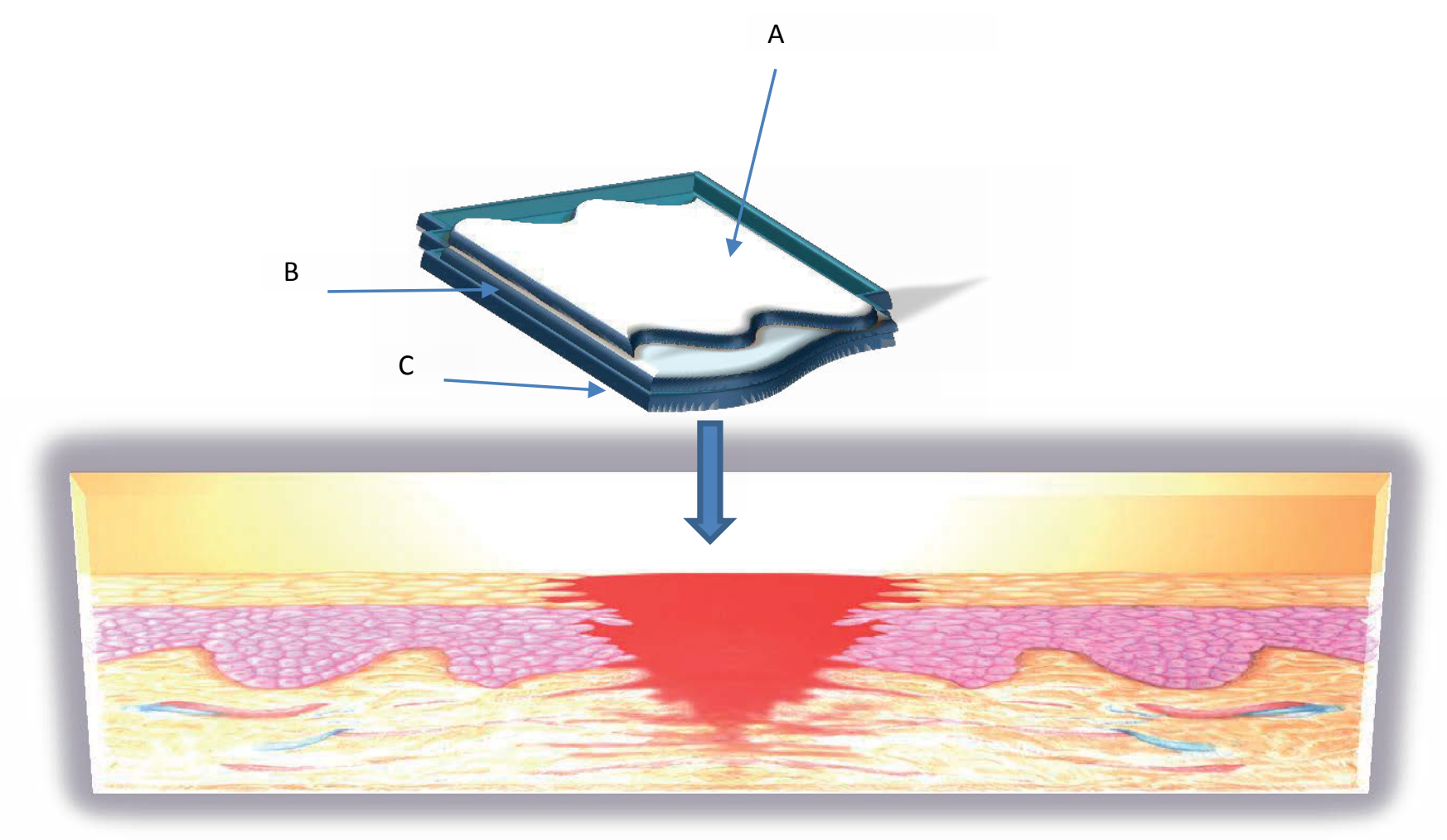

Figure 1

The schematic of multilayered controlled released topical patch on wound A) PVA electro spun layer containing Tetracycline B) PCL electro spun layer C) Chitosan coating.

by immersion in a chitosan solution at $308^{\circ} \mathrm{C}$ for $1 \mathrm{~h}$. For fixation, the chitosan-coated PVA matrix was treated with a $1 \mathrm{~N}$ sodium hydroxide solution after the chitosan coating and followed with by a wash with deionized water until the $\mathrm{pH}$ was neutral. The chitosan-coated PVA/PCL matrix was dried in a vacuum oven at room temperature for $24 \mathrm{~h}$.

\section{Characterization of fibrous scaffold}

Scanning electron microscopy (SEM): The electrospun nanofibers on aluminum foil were coated with a thin layer of gold by a BioRad E5200 auto sputter coater (England). For the morphological observations of the samples, a scanning electron microscope (CamScan MV2300 model, Oxford) with 5000X magnification was used. The mean values of the nanofiber diameters from five different sections were recorded.

Water uptake: The degree of swelling for the nanofiber mats was calculated by equation (1), and tests were carried out in the release medium (acetate buffer solution, $\mathrm{PBS}, \mathrm{pH}=5.5$ ) at $36.5^{\circ} \mathrm{C}$ for 1,6 , and $24 \mathrm{~h}$.

Degree of swelling: $\frac{M-M_{d}}{M_{i}} \times 100$

Where $M$ is the weight of swollen nanofiber samples which were wiped dry with filter paper, $M_{d}$ is the dried mass of immersed sample in Phosphate-buffered saline (PBS), measured by drying the swollen nanofiber mats in an oven at $45^{\circ} \mathrm{C}$ until a constant weight was reached, and $M_{i}$ is the initial dry mass of the sample.

Mechanical properties: The mechanical properties of PVA/
PCL scaffold with and without Chitosan coating were measured using a Tytron 250 Microforce Testing System (MTS Systems, Minneapolis, MN, USA) with a linear DC servomotor. Testing protocols followed the guidelines established in ASTM D 3039/D $3039 \mathrm{M}-00$. The $0.5310 .5 \mathrm{~cm}$ sections of varying thicknesses were sandwiched at their ends between sections of sandpaper, which were then installed onto $5.332 .5 \mathrm{~cm}$ stainless steel spring-loaded grips (MTS Systems). Ends were mounted leaving a gauge length for mechanical loading (portion of sample between grips) at approximately $7 \mathrm{~cm}$, with an intentional stress concentration at the center of the specimen due to the sample. Load-deformation data were recorded for uniaxial testing, at a deforming rate of 10 $\mathrm{mm} / \mathrm{min}$ and value for tensile modulus was determined.

Fourier transforms infrared spectroscopy: Fourier transform infrared (FTIR) spectroscopy was conducted using a Spectrum 100 FTIR spectrometer (PerkinElmer, Massachusetts, USA) fitted with an ATR attachment. The scanning range was $4000-600 \mathrm{~cm}^{-1}$, and the resolution set at $1 \mathrm{~cm}^{-1}$.

\section{In vitro analysis}

In vitro drug release studies: Drug release from the electrospun nanofiber mats were measured by placing a known mass with an approximate dimension $(2.5 \mathrm{~cm} \times 2.5 \mathrm{~cm})$ of material into $20 \mathrm{~mL}$ of sodium acetate buffer at $\mathrm{pH}$ of 5.5 under constant stirring at $36.5^{\circ} \mathrm{C}$. at specific time interval the amount of Tetracycline released to the media calculated with ultravioletvisible spectrometer (UNICAM Series 8700 model, Philips Co., 
Amsterdam, the Netherlands) using the calibration curve with $R^{2}$ value of 0.9962 at the maximum wavelength of $365 \mathrm{~nm}$ for Tetracycline hydrochloride in water.

In vitro antibacterial test: The antibacterial assay of the electrospun samples were determined against a Gram-positive bacterium, Staphylococcus aureus (S. aureus) using the disc diffusion method. This method was performed in a Caso Agar medium solid agar Petri-dish. The multilayered fiber samples were cut into disc shapes $1 \mathrm{~cm}$ in diameter, sterilized under UV light for $2 \mathrm{~h}$ and placed on $\mathrm{S}$. aureus cultured agar plates. Then, they were incubated for $24 \mathrm{~h}$ at $37^{\circ} \mathrm{C}$, and the inhibitory zone was recorded.

\section{Results and Discussion}

The aim of the experiment was focused on the preparation of the nanofiber mats with sustainable antibiotic release properties for topical application.

\section{Characterization of the electrospun scaffold}

We investigated the physicochemical properties of multilayered patch for topical delivery of Tetracycline. The SEM images of PVA and PCL electrospun sheet is shown in (Figure 2). The micrograph of the PVA electrospun sheet containing Tetracycline shows that the concentration of Tetracycline influences the structure of the nanofibers. It should be noted that the 5\% Tetracycline/PVA SEM shows beads, however, the 3\% Tetracycline/PVA contains no beads. As a result this indicates that the 3\% Tetracycline hydrochloride/PVA solution has a suitable compatibility with the polymer solvent system [21].
The SEM results also depict the addition of the Tetracycline decreased the average diameter of nanofibers due to the viscosity reduction of polymeric solutions, as the drug behaves as a plasticizer between the polymer chain layers. Generally, a polymer solution with a higher viscosity during the electrospinning process resists elongation. In other words, viscosity reduction of polymer solution results in better stretching of the injected jet and easier elongation which results to the production of finer fibers. Similarly, in the Zamani et al. [8] study, the addition of Tetracycline also reduced the mean fiber diameter [8]. As it can be seen in (Figure 2), the addition of the PCL layer has decreased the porosity of the mesh of fibers. This could lead to a decrease in permeability and a reduction in water uptake which could consequently allow for a controllable drug release profile.

The water uptake of biomaterial is an important factor for biological and biomedical applications due to its influence on cellular adhesion, proliferation, and growth. We assessed the water uptake of the electrospun scaffolds, (Figure 3 ) shows the degree of swelling in PVA/PCL and PVA/PCL/Chitosan mats in a drug release environment (acetate buffer, $\mathrm{pH}=5.5$ ) at $36.5^{\circ} \mathrm{C}$ for 1,6 , and $24 \mathrm{~h}$. As it can be seen in (Figure 3), the swelling degree of PVA/PCL coated with chitosan increased in comparison to PVA/ $P C L$. The variation in swelling degrees of the two samples is due to the water absorbent properties of chitosan. An increase in swelling rate means the scaffold could keep water during wound healing more efficiently, as a result, making the removal of the wound dressing easier. In addition, the increase in water uptake also improves the oxygen transmission to the wound [22].

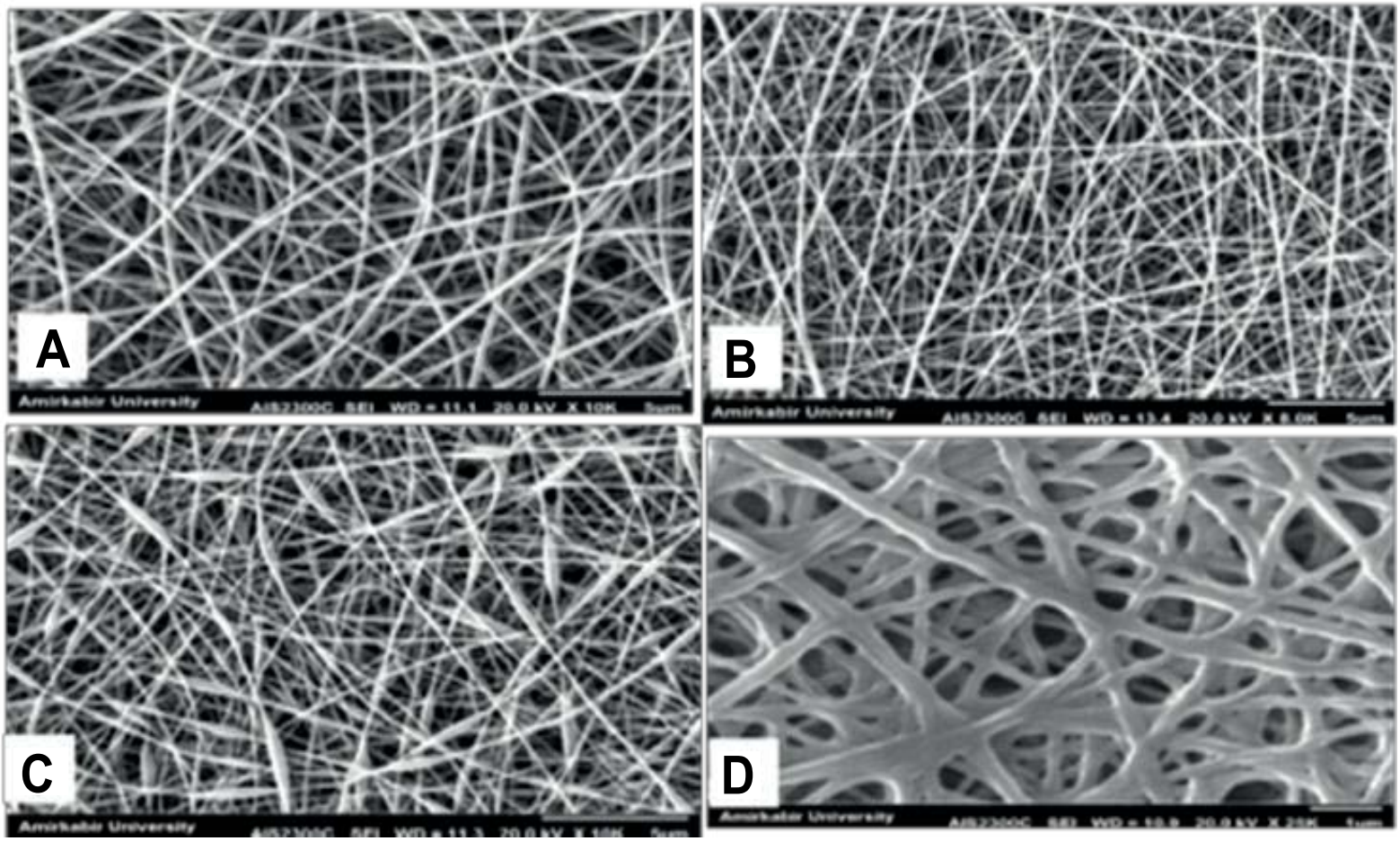

Figure 2 SEM images of the nanofibrous mats prepared from A) PVA 10\% B) PVA 10\%/Tetracycline 5\% C) PVA 10\%/Tetracycline 3\% D) PVA $10 \% / P C L ~ 10 \%$ 


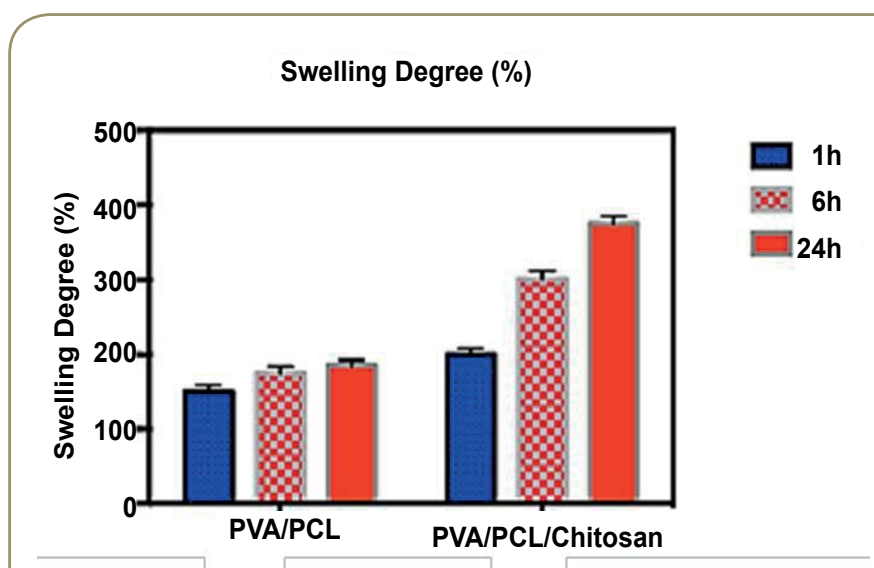

Figure 3 Swelling degree of PVA/PCL and PVA/PCL/Chitosan after 1,6 and $24 \mathrm{~h}$.

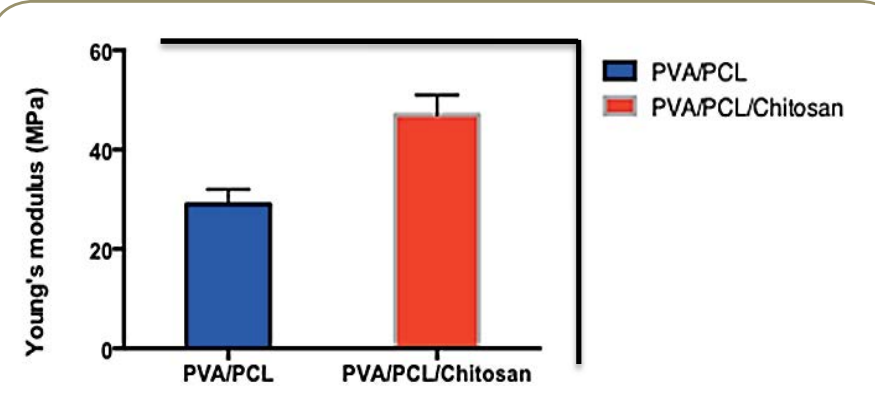

Figure 4

Comparison diagram of the young modulus of $\mathrm{PVA} / \mathrm{PCL}$ and PVA/PCL/Chitosan.

The mechanical properties of PVA/PCL scaffold before and after the addition of chitosan are represented in (Figure 4). The elastic modulus of PVA/PCL scaffold was increased by the addition of chitosan. The tensile strength of PVA/PCL/Chitosan increased significantly due to the closure of empty spaces in the pores of $\mathrm{PCL}$, and thereby improving film tensile properties and water vapor permeability.

FTIR spectroscopy was used to evaluate the possible changes in materials during the fabrication process (Figure 5) depicts the five spectra produced for PVA, Tetracycline, $\mathrm{PCL}$, chitosan, and PVA+Tetracycline/PCL/chitosan. PVA detection is possible by three bands corresponding to $\mathrm{OH}$ at $3422 \mathrm{~cm}^{-1}, \mathrm{CH}_{2}$ at 2800 and $2900 \mathrm{~cm}^{-1}$ and aliphatic stretching at 1380 and 1448 , for PCL the most important band is for carbonyl group at 1727 and also at 2800 and $2900 \mathrm{~cm}^{-1}$ we have $\mathrm{CH}_{2}$ stretching and at $1075 \mathrm{~cm}^{-1} \grave{\mathrm{C}}-\mathrm{O}$ stretching. Tetracycline showed a strong band at $1672 \mathrm{~cm}^{-1}$ for the carbonyl group , amidic and $\mathrm{NH}_{2}$ stretching at $3000-3400 \mathrm{~cm}^{-1}$, in addition there was $\mathrm{C}=\mathrm{C}$ stretching at $1527 \mathrm{~cm}^{-1}$ and $1583 \mathrm{~cm}$ 1 , and $2800-2900 \mathrm{~cm}^{-1} \mathrm{CH}_{2}$ aliphatic stretching. For chitosan, the $\mathrm{NH}_{2}$ peak was overlapped by the $\mathrm{OH}$ group and at $1075 \mathrm{~cm}^{-1}$ there was $\mathrm{C}-\mathrm{O}$ stretching.

The FTIR of PVA/PCL/Chitosan with Tetracycline showed that $\mathrm{NH}_{2}$ group of Tetracycline was overlapped by the $\mathrm{OH}$ group of polymers and $\mathrm{OH}$ group of PVA intensify the $\mathrm{OH}$ peak of PCL. Also a $1732 \mathrm{~cm}^{-1}$ peak showed the carbonyl group of $\mathrm{PCL}$ and 1583 $\mathrm{cm}^{-1} \mathrm{C}=\mathrm{C}$ stretching showed the Tetracycline group.
The FTIR spectra of PVA/PCL/Chitosan did not show any difference in their absorbance pattern. This observation confirmed that the chemical composition of the scaffolds did not change during the fabrication process.

\section{In vitro drug release study}

Figure 6 shows the accumulative release of Tetracycline from $\mathrm{PVA} / \mathrm{PCL}$ and PVA/PCL/chitosan in an acetate buffer with $\mathrm{pH} 5.5$ for a period of $168 \mathrm{~h}$ at $36.5^{\circ} \mathrm{C}$. As it is shown in (Figure 6) the drug release rate profiles of $\mathrm{PVA} / \mathrm{PCL} / \mathrm{Chitosan}$ have a bimodal behavior and contain a burst release phase in which $69 \%$ of loaded Tetracycline released before $5 \mathrm{~h}$, and then sustained release phase started for $168 \mathrm{~h}$ and the remain loaded drug released.

The drug release profile from a polymer matrix can be explained by the Fickian diffusion mechanism which introduces the drug penetration as the most important factor in drug release. Other mechanisms can be applied for the rate of drug release such as, the release from the surface of the nanofibrous mats, or the degradation of the polymer matrix [23].

According to the release profile of our samples, the burst release of Tetracycline in the beginning is attributed to the diffusion of the drug located near the surface, and the penetration of Tetracycline through PCL electrospun layer. The PCL layer acted as the diffusion barrier in the drug penetration route, and by coating the electrospun bilayer with chitosan, we could achieve a controllable release profile. As it was shown in (Figure 6), the all of the loaded drug released from PVA/PCL within $20 \mathrm{~h}$. However, the PVA/PCL/Chitosan demonstrated a sustained release pattern with the duration of $168 \mathrm{~h}$. In the first $5 \mathrm{~h} 69 \%$ of loaded antibiotic was released and the remaining drug was gradually released due to the continuous degradation of the nanofibers.

In vitro antibacterial test: The relative antibacterial activity of PVA/PCL and PVA/PCL/Chitosan nanofibrous mats containing Tetracycline was evaluated qualitatively on $S$. aureus and results are presented in (Figure 7). Based on the size of inhibition zone, $\mathrm{PVA} / \mathrm{PCL} / \mathrm{Chitosan}$ had stronger antibacterial effect in compare to PVA/PCL which could be related to chitosan antibacterial effect.

According to the study done by Govender et al., the minimum inhibitory concentration for Tetracycline is $1 \mu \mathrm{g} / \mathrm{cc}$ in PBS with acidic $\mathrm{pH}$ [24]. Similarly, the in vitro release test results also showed the concentration of drug released at each time point throughout the 168 hours test period was above $1 \mu \mathrm{g} / \mathrm{mL}$ and in the range of 6.82-24.07 $\mu \mathrm{g} / \mathrm{cc}$ for PVA/PCL/Chitosan/Tetracycline. In another study, single dose topical administration of control release Cefazolin microspheres was effective for the treatment of MRSA in high risk of infection within and around the surgical wound [4] thus it could be an effective approach to treat wound infection with control released drug delivery system.

\section{Conclusion}

The prepared multilayered wound dressing provides a sustainable release of Tetracycline for one week with the appropriate physicochemical properties. In this system fluctuating in drug level would be prevented which lead to the decrease chance of appearing resistance organism. In addition, Chitosan coating increase the in vitro antibacterial activity of the system along the improvement in mechanical properties and in vitro drug release. 

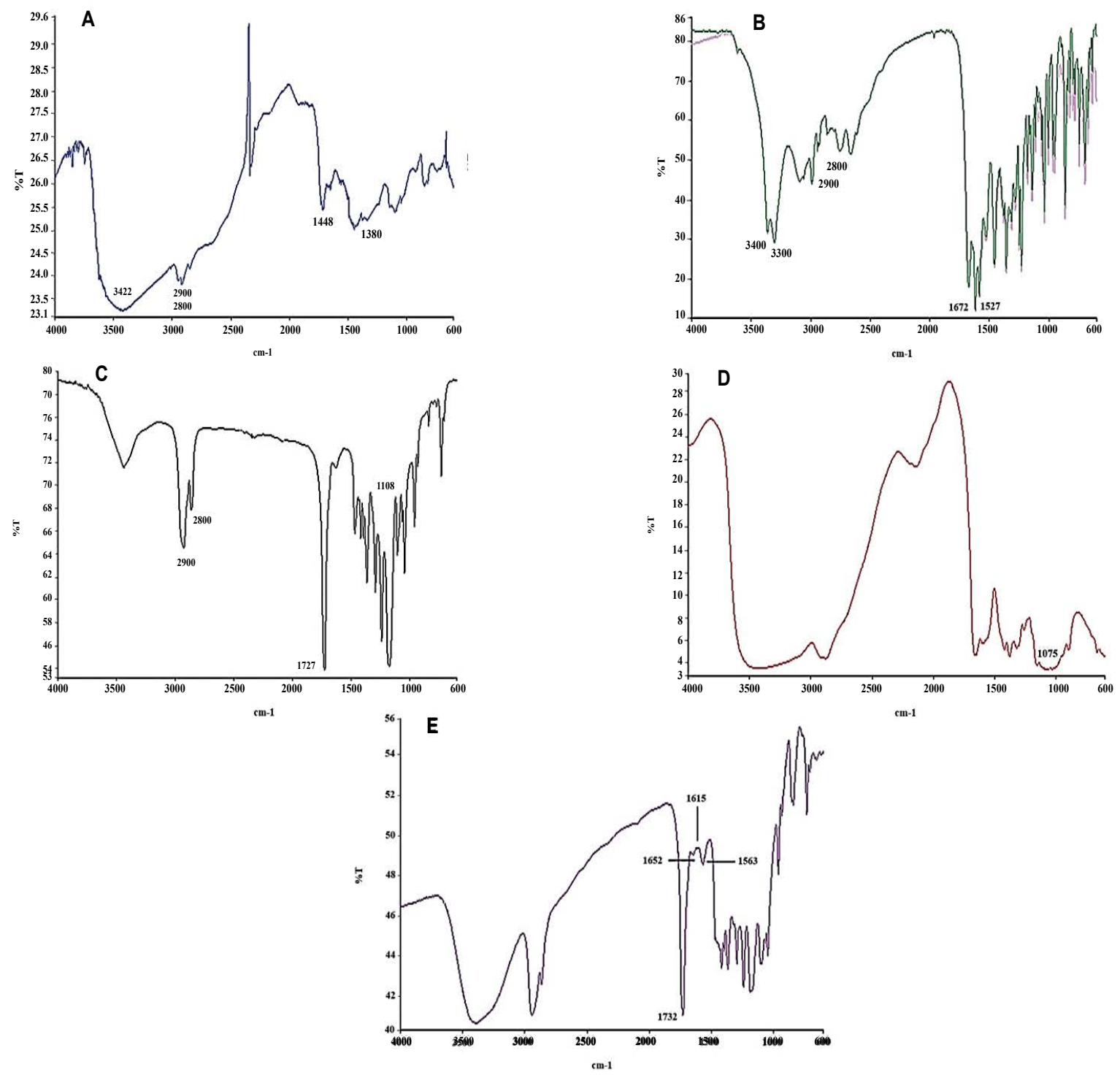

Figure 5 FTIR spectrums of a) PVA b) Tetracycline c) PCL d) Chitosan e) PVA+Tetracycline/PCL/Chitosan.

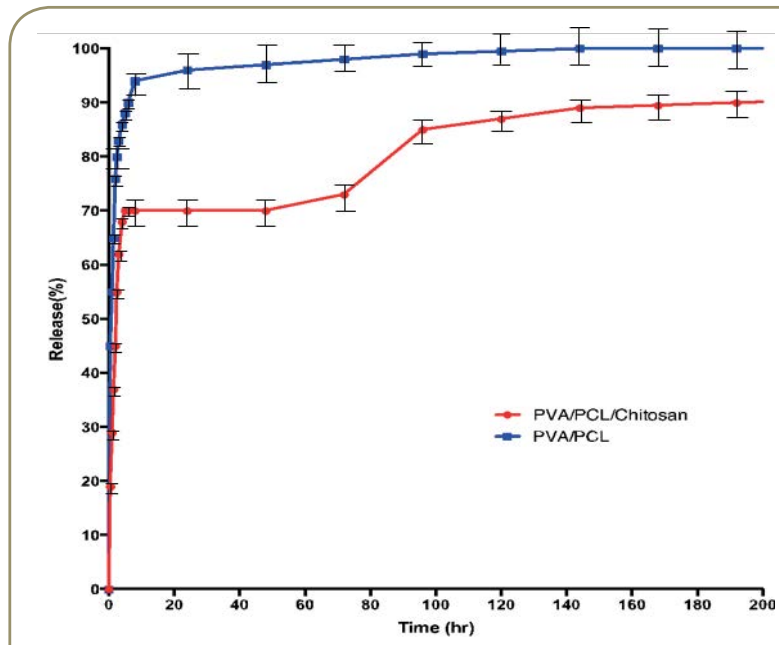

Figure 6 Release profiles of Tetracycline release from PVA/PCL and $\mathrm{PVA} / \mathrm{PCL} / \mathrm{Chitosan}$ as a function of time at $37^{\circ} \mathrm{C}$ in PBS with $\mathrm{pH}$ 5.5.

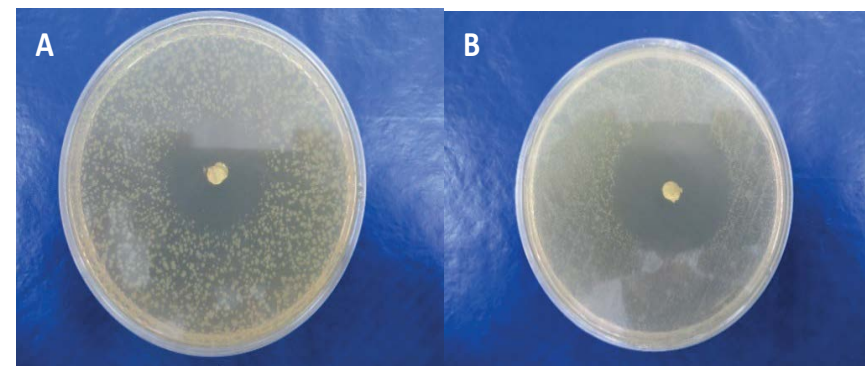

Figure 7 Agar antibacterial plates on $S$. aureus after $24 \mathrm{~h}$ of incubation with A) PVA+Tetracycline/PCL and B) PVA+Tetracycline/PCL/Chitosan. 


\section{References}

1 Mayon-White RT, Ducel G, Kereselidze T, Tikomirov E (1988) An international survey of the prevalence of hospital-acquired infection. Journal of Hospital Infection 11: 43-48.

2 Siddiqui AR, Bernstein JM (2010) Chronic wound infection: facts and controversies. Clinics in dermatology Jan 28: 519-526.

3 Bickers DR, Lim HW, Margolis D, Weinstock MA, Goodman C, et al. (2006) The burden of skin diseases: 2004 a joint project of the American Academy of Dermatology Association and the Society for Investigative Dermatology. Journal of the American Academy of Dermatology 55: 490-500.

4 Fallon MT, Shafer W, Jacob E (1999) Use of cefazolin microspheres to treat localized methicillin-resistant Staphylococcus aureus infections in rats. The Journal of surgical research 86: 97-102.

5 Craig WA (2003) Basic pharmacodynamics of antibacterial with clinical applications to the use of beta-lactams, glycopeptides and linezolid. Infectious disease clinics of North America 17: 479-501.

6 Levison ME, Levison JH (2009) Pharmacokinetics and pharmacodynamics of antibacterial agents. Infectious disease clinics of North America 23: 791-815.

7 Megelski S, Stephens JS, Chase DB, Rabolt JF (2002) Micro- and Nano-structured Surface Morphology on Electrospun Polymer Fibers. Macromolecules American Chemical Society 35: 8456-8466.

8 Zamani M, Morshed M, Varshosaz J, Jannesari M (2010) Controlled release of metronidazole benzoate from poly $\varepsilon$-caprolactone electrospun nanofibers for periodontal diseases. European Journal of Pharmaceutics and Biopharmaceutics 75: 179-85.

9 Liang D, Hsiao BS, Chu B (2007) Functional electro spun nanofibrous scaffolds for biomedical applications. Advanced Drug Delivery Reviews 59: 1392-1412.

10 Khil MS, Cha DI, Kim HY, Kim IS, Bhattarai N, et al. (2003) Electro spun nanofibrous polyurethane membrane as wound dressing. Journal of biomedical materials research Part B, Applied biomaterials 67: 675-679.

11 Wu M, Bao B, Yoshii F. Makuuchi KIrradiation of cross-linked, poly (vinyl alcohol) blended hydrogel for wound dressing. Journal of Radioanalytical and Nuclear Chemistry, Kluwer Academic Publishers 250: 391-395.

12 Queen D, Evans JH, Gaylor JDS, Courtney JM, Reid WH (1987) Burn wound dressings - a review. Burns 13: 218-228.
13 Jachuck SJ, Bound CL, Steel J, Blain PG (1989) Occupational hazard in hospital staff exposed to 2 percent glutaraldehyde in an endoscopy unit. Occupational Medicine 39: 69-71.

14 Takigawa T, Endo Y (2006) Effects of glutaraldehyde exposure on human health. Journal of Occupational Health 48: 75-87.

15 Moats WA. Inactivation of antibiotics by heating in foods and other substrates - A Review. International Association for Food Protection.

16 Dash TK, Konkimalla VB (2012) Poly-€-caprolactone based formulations for drug delivery and tissue engineering: A review. Journal of controlled release official journal of the Controlled Release Society 158: 15-33.

17 Zahedi P, Karami Z, Rezaeian I, Jafari SH, Mahdaviani P, et al. (2012) Preparation and performance evaluation of Tetracycline hydrochloride loaded wound dressing mats based on electro spun nanofibrous poly(lactic acid)/poly( $\epsilon$-caprolactone) blends. Journal of Applied Polymer Science. 124: 4174-4183.

18 Kang YO, Yoon IS, Lee SY, Kim DD, Lee SJ, et al. (2010) Chitosan-coated poly(vinyl alcohol) nanofibers for wound dressings. Journal of Biomedical Materials Research Part B, Applied Biomaterials 92: 568-576.

19 Ueno H, Mori T, Fujinaga T (2001) Topical formulations and wound healing applications of chitosan. Advanced Drug Delivery Reviews 52: 105-115.

20 Kenawy ER, Bowlin GL, Mansfield K, Layman J, Simpson DG, et al. (2002) Release of Tetracycline hydrochloride from electro spun poly(ethylene-co-vinylacetate), poly(lactic acid), and a blend. Journal of Controlled Release 81: 57-64.

21 Zeng J, Yang L, Liang Q, Zhang X, Guan H, et al. (2005) Influence of the drug compatibility with polymer solution on the release kinetics of electro spun fiber formulation. Journal of Controlled Release: Official journal of the Controlled Release Society 105: 43-51.

22 Mi FL, Wu YB, Shyu SS, Schoung JY, Huang YB, et al. (2002) Control of wound infections using a bilayer chitosan wound dressing with sustainable antibiotic delivery. Journal of biomedical materials research 59: 438-449.

23 Soares JS, Zunino P (2010) A mixture model for water uptake, degradation, erosion and drug release from polydisperse polymeric networks. Biomaterials 31: 3032-3042.

24 Govender S, Pillay V, Chetty DJ, Essack SY, Dangor CM, et al. (2005) Optimisation and characterisation of bioadhesive controlled release Tetracycline microspheres. International journal of pharmaceutics 306: 24-40. 